\title{
The prevalence of undiagnosed abnormalities on non-contrast-enhanced computed tomography compared to contrast-enhanced computed tomography of the brain
}

\begin{abstract}
Authors:
Cornelia Minné

Margaret E. Kisansa ${ }^{1}$

Nazeema Ebrahim ${ }^{2}$

Farhana E. Suleman ${ }^{3}$

Nonjabulo Z. Makhanya ${ }^{3}$

Affiliations:

${ }^{1}$ Department of Diagnostic

Radiology, University of

Limpopo, South Africa

${ }^{2}$ Department of Radiography,

University of Limpopo,

South Africa

${ }^{3}$ Department of Radiology, University of Pretoria,

South Africa

\section{Correspondence to:}

Cornelia Minné

\section{Email:}

riaminne@gmail.com

Postal address:

PO Box 100143, Moreletta

Plaza 0167, South Africa

Dates:

Received: 08 May 2013

Accepted: 18 Jan. 2014

Published: 01 Aug. 2014

How to cite this article: Minné $\mathrm{C}$, Kisansa ME, Ebrahim N, Suleman FE, Makhanya NZ. The prevalence of undiagnosed abnormalities on noncontrast-enhanced computed tomography compared to contrast-enhanced computed tomography of the brain. $S$ Afr J Rad. 2014;18(1); Art. \#598, 7 pages. http://dx.doi. org/10.4102/sajr.v18i1.598
\end{abstract}

Background: Even though magnetic resonance imaging (MRI) is the gold standard investigation for intracranial pathology, it is not widely available in developing countries and computed tomography (CT) of the brain remains the first-line investigation for patients with suspected intracranial pathology. It is generally accepted that certain intracranial pathology can be missed on non-contrast-enhanced CT (NECT) of the brain if a contrast-enhanced CT (CECT) is not done. We have to consider on the one hand the risk of delayed or missed diagnosis and on the other hand the cost, increased radiation exposure and contrast-induced reactions. Advances in CT technology have also improved the resolution of CT scan images, making it easier to identify pathology on an NECT of the brain. To date, no study comparing NECT to CECT of the brain, utilising 64-slice CT technology, has been published.

Objectives: To determine the prevalence of undiagnosed abnormalities on non-contrastenhanced computed tomography (NECT) scans of the brain reported as normal, on a 64-slice CT scanner.

Method: A descriptive retrospective study was undertaken of CT brain scans done during a 12-month period at a tertiary provincial hospital in the Northern Tshwane district of Gauteng, South Africa. The CT brain scans were evaluated by three reviewers (general radiologists). The NECT and contrast-enhanced computed tomography (CECT) scans of the brain were reviewed independently on separate occasions. Reviewers were blinded to patient history, each other's interpretation, and to their own interpretation of the NECT when evaluating the CECT and vice versa. Discrepancies in interpretation were resolved during a consensus meeting between all three reviewers. The reviewers also re-evaluated the NECT scans of the cases with undiagnosed abnormal findings during this session. A decision was made pertaining to the visibility of the abnormal findings on the NECT scan.

Results: In this study, 3.28\% of cases had abnormal findings undiagnosed by three reviewers on the NECT scans. Re-evaluation by the panel reduced this to $1.42 \%$, indicating a reading error of $1.85 \%$.

Conclusion: There is a small prevalence of missed abnormal findings on the NECT scan when using only NECT. Omitting unnecessary CECT will reduce the radiation exposure to the patient and reduce the risk of adverse events from the use of intravenous iodinated contrast. Alternatively, doing only a CECT scan would reduce the risk of missing abnormal findings and would also decrease the patient's exposure to radiation.

\section{Introduction}

We live in a technologically advanced age, but not in an ideal world. Magnetic resonance imaging (MRI) is the gold standard investigation for intracranial pathology, but is not widely available in developing countries. ${ }^{1}$ Within the public health sector of South Africa, MRI is limited to a few tertiary institutions. Radiologists and clinicians in many centres must therefore rely on computed tomography (CT) of the brain as a first-line investigation for patients with suspected intracranial pathology. Traditionally these patients would be investigated with non-contrast-enhanced CT (NECT) followed by contrast-enhanced CT (CECT) of the brain.

Most pathological processes result in alterations in the blood-brain barrier that lead to local oedema and contrast enhancement. ${ }^{1,2,3,4}$ Imaging characteristics, including enhancement patterns, have been described for various pathological conditions. ${ }^{1,2,3,5}$ It is well recognised that vascular 
abnormalities and isodense lesions could be undiagnosed on NECT and that CECT may subsequently demonstrate these lesions. $^{2}$ A CECT can also confirm, change or shorten the differential diagnosis. ${ }^{1,3}$

Important factors to consider before subjecting patients to CECT are:

- the risk of adverse events after intravenous injection of iodinated contrast media

- the cost and time implication of doing an NECT and CECT of the brain

- the radiation dose to the patient.

On the other hand, performing only an NECT poses a risk of missing abnormal findings that a CECT could detect. The omission of a CECT may lead to misdiagnosis or delayed diagnosis in patients with possible intracranial pathology.

Existing policies for the use of CECT were established in the 1970s based on experiences with early-generation CT scanners. ${ }^{3,6}$ Barrington et al. regarded CECT as unhelpful if the NECT was normal. ${ }^{7}$ Later studies by Chishti et al. ${ }^{1}$ and Huckman $^{2}$ demonstrated that pathology undiagnosed on NECT could subsequently be seen on CECT. Other authors suggested the use of contrast media in patients with normal NECT scans if there were focal clinical signs, ${ }^{1,3,6}$ whilst some like Butler et al. were of the opinion that a CECT on its own was as accurate as an NECT and a CECT combined. ${ }^{8}$ We have to consider that improved CT scanners with better resolution may mean that increased pathology would be detected on the NECT and could eliminate the need for performing a CECT of the brain.

To date, no study comparing NECT to CECT of the brain, utilising 64-slice CT technology, has been published.

\section{Aim}

The aim of this study was to determine the prevalence of occult abnormalities on an NECT of the brain (done on a 64-slice CT scanner), if a CECT was omitted.

\section{Method}

Ethics approval was obtained from the Medunsa Campus Research and Ethics Committee (MREC) of the University of Limpopo. A descriptive retrospective study was undertaken of patients who presented for a CT of the brain to the diagnostic radiology department of Dr George Mukhari Hospital, a tertiary provincial hospital in the Northern Tshwane district of Gauteng province, South Africa. Patient confidentiality was maintained during the study. All patients underwent an NECT directly followed by a CECT of the brain. Patients selected for the study were imaged during the period 01 October 2007 - 30 September 2008. CT brain scans of all patients suspected of having intracranial pathology, referred for brain imaging, were considered. Patients were excluded if they had an acute head injury, an abnormal NECT of the brain, an incomplete CT study, or if severe artefacts were present on the $\mathrm{CT}$ images.
All brain CT scans were performed on a Toshiba Aquilion model TSX-101A, a 64 multislice CT scan system. CT scans were viewed on a $1600 \times 1200$ pixel monitor and a high-definition video-card workstation. CT scans were evaluated by three reviewers (general radiologists). None of the reviewers were neuroradiologists. All CT scans were interpreted by the reviewers in the radiology department during working hours, simulating normal working conditions. Reviewers were blinded to their own interpretation of the NECT when the CECT was evaluated and vice versa. Reviewers were also blinded to each other's interpretation and to patient history. Only intracranial structures were evaluated; bony abnormalities and abnormal findings outside the skull were disregarded. The NECT and CECT were evaluated independently on separate occasions and separate datasheets were completed and then captured as follows: If two or three reviewers interpreted the scan as abnormal it was entered as 'abnormal'. If only one of the three reviewers interpreted a scan as abnormal, the scan was entered as 'undetermined'. All three reviewers had to agree that a scan was normal before it was entered as 'normal'. This process was done for both NECT and CECT. Discrepancies in interpretation were then resolved during a consensus meeting between all three reviewers. At this meeting reviewers also re-evaluated the cases with abnormal findings on CECT that were undiagnosed on the NECT, and a decision was made as to the visibility of the abnormal findings on the NECT. Both the NECT and CECT were reviewed consecutively. The reviewers were blinded to the history of the patient during the consensus meeting. The data was then updated. The records of cases with an undiagnosed abnormal finding on NECT were reviewed and summarised.

\section{Statistics}

Sample size was calculated assuming that a proportion of 0.4 brain NECT would be normal scans and the prevalence of undiagnosed abnormalities on NECT as compared to CECT would be 0.05 . A significance threshold of 0.05 was selected. A minimum sample of $521 \mathrm{CT}$ scans was required. The number of normal, abnormal and undetermined outcomes were counted within each group, using the Statistical Analysis System (SAS) (2010). A finding was regarded as undiagnosed if the consensus decision on the NECT was normal and the CECT abnormal. Inter-rater agreement was assessed with Kappa statistics using Medcalc (2011).

\section{Results}

A total of 992 patients were initially selected by the lead researcher. Cases with subtle abnormalities were included in the initial selection to reduce selection bias. Subsequent evaluation by all three reviewers excluded patients with subtle abnormalities on NECT, reducing the sample size to 726. The gender distribution was more or less equal. Age categories are summarised in Figure 1. The majority of patients were between the ages of 1 and 39, with the largest group 30-39 years (139), the second largest group 1-9 years (127) and the third largest group 20-29 years (120). 
Kappa statistics were performed between the reviewers on the CECT dataset before consensus was reached (Table 1). The inter-rater agreement between reviewer 1 and 3 was moderate but between reviewer 2 and other reviewers fair.

After the three reviewers reached consensus, the data was updated. Twenty-five cases were excluded as they were regarded to have an abnormal NECT. The number of patients with normal NECT was thus reduced to 701. Of the 701 patients that had a normal NECT, 23 (3.28\%, CI 1.98-4.88\%, $p<0.001)$ cases had abnormalities on the CECT.

The 23 cases with abnormal findings undiagnosed on the NECT were re-evaluated by the three reviewers during the consensus meeting. Thirteen $(1.85 \%)$ of these cases had an identifiable abnormal finding on the NECT in retrospect and 10 cases $(1.42 \%)$ were still deemed normal on NECT.

Abnormalities detected on CECT that were not detected by the NECT are summarised in Table 2. Patient hospital records of only 15 of the 23 cases with abnormal findings undiagnosed on NECT were available from the hospital archives. Nine of these 15 cases had a history or clinical finding that would alert the radiologist to proceed with a CECT. Two cases had recorded fever, three had focal signs, three had confusion, three had meningism, three had convulsions and one had a recorded history of dehydration that poses a risk for dural venous sinus thrombosis.

\section{Discussion}

A CECT contributed extra information in 3.28\% (23 out of 701 cases) of normal NECT of the brain. Retrospectively only $1.42 \%$ (10 out of 701 cases) had abnormal findings on CECT that were not visible on the NECT scans. The difference between $3.28 \%$ and $1.42 \%$ constitutes a reader error of $1.85 \%$ (13 out of 701 cases).

The abnormalities undiagnosed by the reviewers in this study (Table 2) can be classified into four main groups:

- Infective: meningitis (Figure 2), empyema (Figure 3)

- Vascular: dural venous sinus thrombosis and vascular malformations (Figure 4)

- Neoplasms: meningioma (Figure 5), pituitary lesions

- Ischaemic events: lacunar infarcts.

When records of cases with an undiagnosed finding were evaluated, it revealed that the majority of these patients had a history or clinical findings that would have guided the radiologist to perform a CECT.

In some cases, a reviewer reported the NECT study as abnormal, but after the administration of contrast media reported it as normal. This demonstrates that in some cases

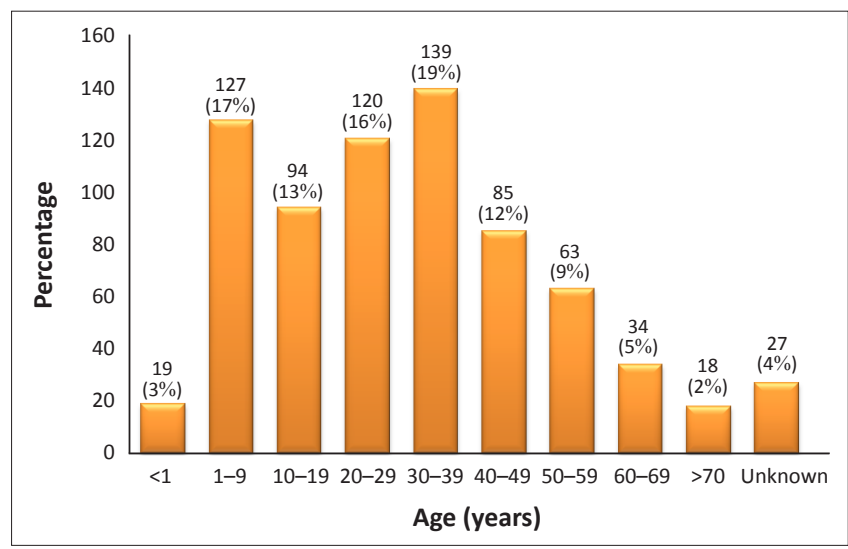

FIGURE 1: Summary of age distribution.

TABLE 1: Summary of Kappa statistics of the reviewers done on the CECT dataset before consensus was reached to determine inter-rater agreement.

\begin{tabular}{llllllll}
\hline Raters & Agree normal & Agree abnormal & Disagree normal & Disagree abnormal & Kappa value & Standard error & 95\% Cl \\
\hline 1 vs 2 & 689 & 6 & 28 & 3 & 0.265 & 0.089 \\
1 vs 3 & 678 & 21 & 13 & 14 & 0.589 & $0.0903-0.439$ \\
2 vs 3 & 688 & 6 & 3 & 29 & 0.258 & $0.4480-0.730$ & $0.0869-0.429$ \\
\hline
\end{tabular}

CECT, contrast-enhanced computed tomography; $\mathrm{Cl}$, confidence interval.

TABLE 2: Summary of findings undiagnosed on NECT.

\begin{tabular}{|c|c|c|c|}
\hline Classification of findings & Presumed diagnosis & $\begin{array}{l}\text { All cases with a finding undiagnosed on } \\
\text { NECT }\end{array}$ & $\begin{array}{l}\text { Cases with the finding not visible on NECT } \\
\text { retrospectively }\end{array}$ \\
\hline \multirow[t]{2}{*}{ Infective } & Meningeal enhancement & $7 \dagger$ & $4 \dagger$ \\
\hline & Empyema/Abscess & 1 & - \\
\hline \multirow[t]{2}{*}{ Vascular } & Arteriovenous malformation & 1 & - \\
\hline & Dural venous sinus thrombosis & 2 & 2 \\
\hline Ischaemic & Lacunar infarct & 2 & - \\
\hline \multirow[t]{3}{*}{ Neoplastic } & Meningioma & 2 & 1 \\
\hline & Pituitary mass & $1 \dagger$ & - \\
\hline & Pituitary microadenoma & 2 & 1 \\
\hline \multirow[t]{4}{*}{ Other } & Pituitary stalk thickened & 1 & - \\
\hline & Enhancing nodule(s) & 3 & 3 \\
\hline & Neurocystisercosis & 1 & - \\
\hline & Middle cranial fossa lesion & 1 & - \\
\hline Total & - & 24 & 11 \\
\hline
\end{tabular}

NECT, non-contrast-enhanced computed tomography.

$\dagger$, One case had dual pathology: Meningitis and a pituitary mass. 

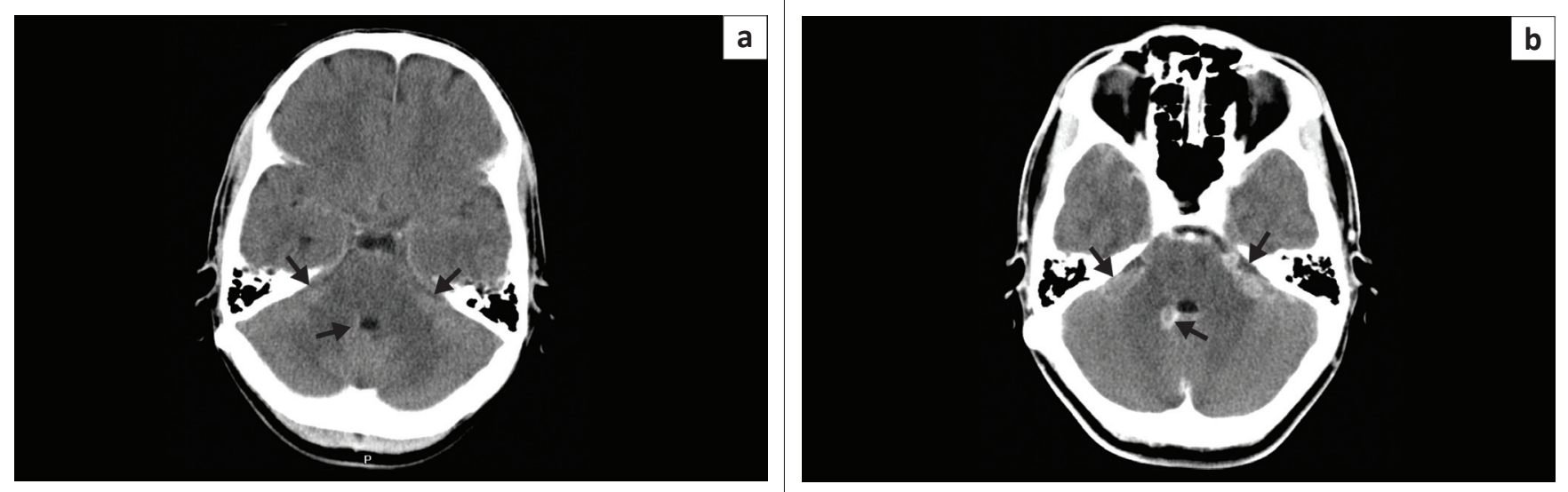

NECT, non-contrast-enhanced computed tomography; CECT, contrast-enhanced computed tomography.

FIGURE 2: (a) NECT and (b) CECT of the cerebellum. Multiple enhancing nodules seen in the CP angle and to the right of the fourth ventricle on the CECT (b, arrows). These nodules are vaguely visible on the NECT (a, arrows) but were missed by the reviewers.
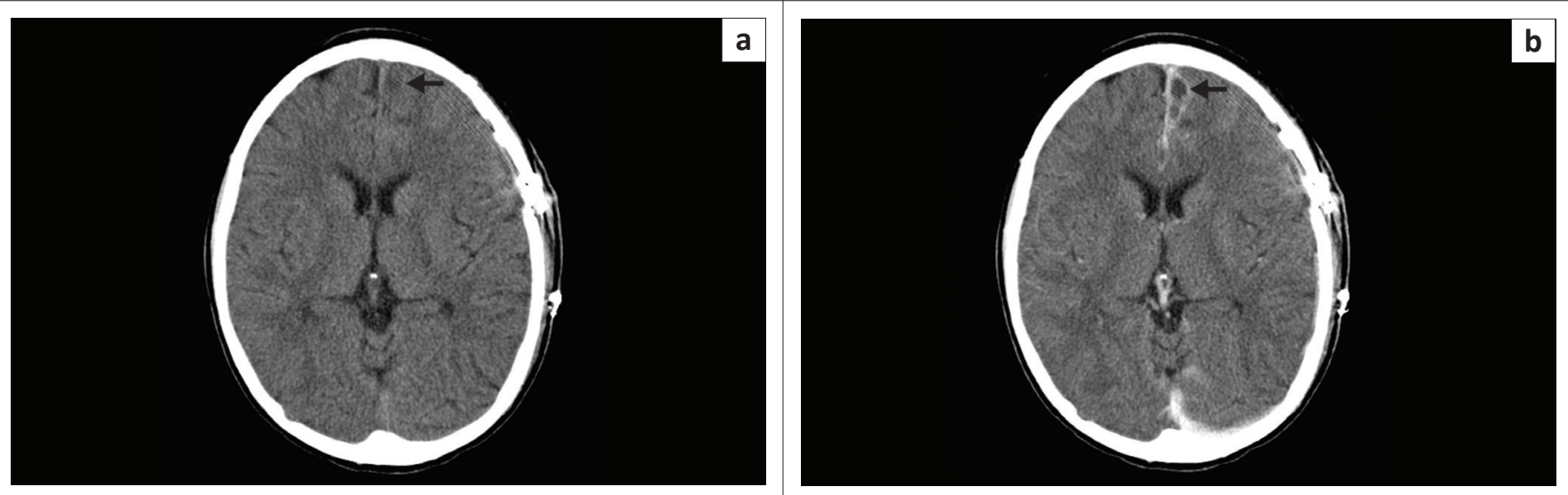

NECT, non-contrast-enhanced computed tomography; CECT, contrast-enhanced computed tomography.

FIGURE 3: (a) NECT and (b) CECT of the brain. The NECT shows a vague parafalx hypodensity (arrow). This finding was undiagnosed during the first review and only identified on the CECT, which shows a small empyema (arrow).
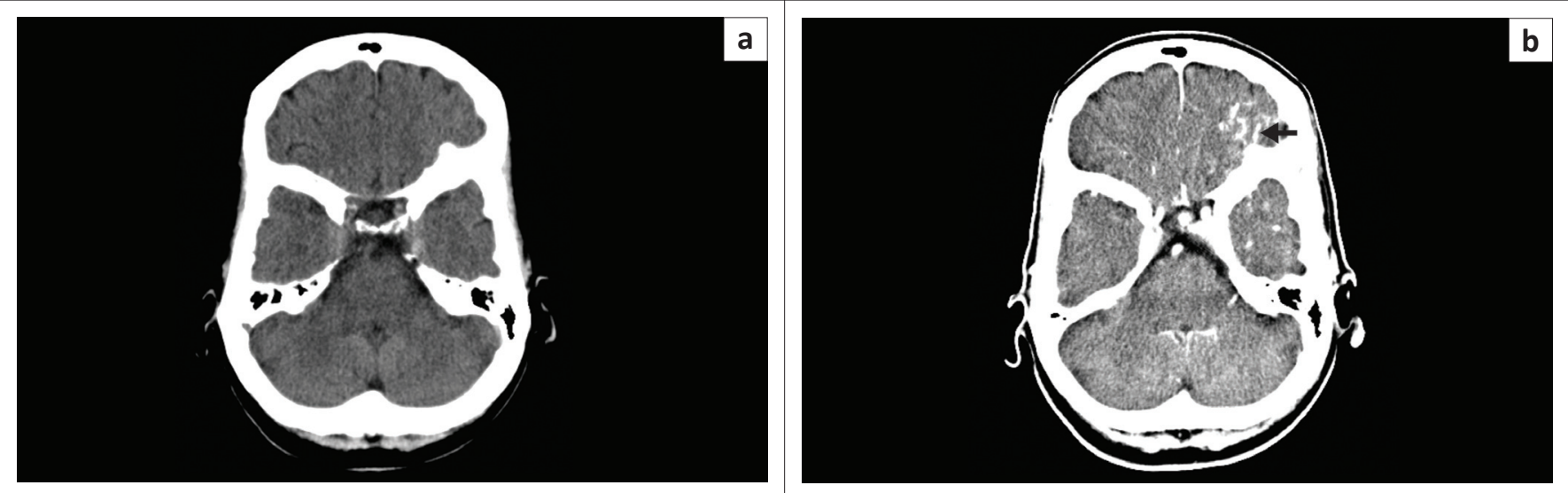

NECT, non-contrast-enhanced computed tomography; CECT, contrast-enhanced computed tomography.

FIGURE 4: (a) NECT and (b) CECT of the brain. No abnormal finding is visible on the NECT but the CECT reveals a vascular abnormality left frontally (arrow).

contrast media will help to eliminate false positive reports and help to confirm a normal brain CT in an equivocal NECT. Chishti et al. ${ }^{1}$ also showed that a CECT can help reduce false positive findings on an NECT.
Undiagnosed findings on NECT may be attributed to several factors. These factors can be divided into imaging factors and reviewer factors. Imaging factors include the quality of the CT images, the density and size of the abnormal findings 


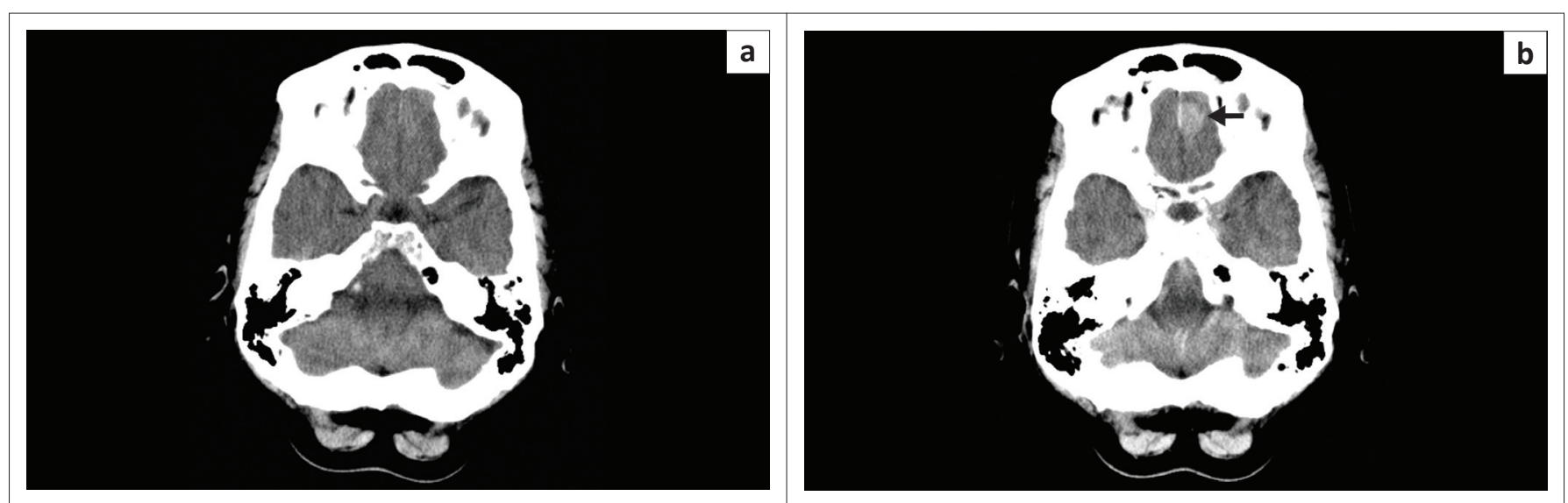

NECT, non-contrast-enhanced computed tomography; CECT, contrast-enhanced computed tomography.

FIGURE 5: (a) NECT and (b) CECT of the brain. The NECT is apparently normal but the CECT demonstrates a small well-defined enhancing lesion (arrow). Meningioma was reported the most likely diagnosis.

and the presence or absence of secondary signs. Pathology will not be visible on an NECT when it is isodense to the surrounding parenchyma and when there are no secondary signs like oedema or mass effect. Reviewer factors can be cognitive or perceptive errors. Cognitive errors occur when the radiologist sees an abnormal finding but interprets it as a normal variant or a normal structure. Perceptive reviewer errors occur when the abnormal finding is overlooked by the reviewer. Perceptive errors are four times more common than cognitive errors. ${ }^{9,10}$ Errors can also be classified into false positive and false negative errors. ${ }^{9,10}$ False negative errors occur five times more commonly than false positive errors.

There are many other factors that influence the accuracy of a radiologist's report. Some of these factors are fatigue, interruptions, incomplete or inaccurate clinical history, unavailability of previous investigations for comparison, poor viewing conditions and poor quality examination., ${ }^{9,10}$ In this study, factors that could have affected the reviewers were: repeated interruptions, fatigue from reading multiple brain CT scans in short periods of time, long periods between reading sessions due to workload, shortage of staff and shortage of workstations. However, these factors are present in most departments and work environments in South Africa.

Our selection criteria may have created a bias, as convenience sampling was done. No history was available to the reviewers as a clinical history would have created an interpretive bias. NECT and CECT were read at different times in order to reduce a possible bias being created by reading two similar CT scans directly after each other. This also prevented the reviewers from reviewing their first evaluation of the NECT and making changes on the datasheet.

CT technology has changed dramatically in the last few years. CT scanners offer increased resolution, decreased radiation, multiplanar imaging and post-reconstruction processing functions. The most recent study by Chishti et al. ${ }^{1}$ investigating the usefulness of intravenous iodinated contrast media in cranial CT from 1997 to 2001 had a total of 547 adult patients with CT scans performed on a third-generation
CT scanner. The patients were divided into three groups. The first group of 496 patients had a normal NECT and no clinical indication for CECT, for example lateralising signs. In this group, only one patient $(0.2 \%)$ had an abnormal CECT (a meningioma). In the second group of 16 patients with lateralising signs, only two patients $(12.5 \%)$ had a normal NECT and an abnormal CECT (meningeal enhancement). In the third group of 35 patients ( 30 abnormal and 5 equivocal cranial NECT), the CECT changed the diagnosis in 12 cases and added to the differential diagnosis in 7 cases. Overall, in this study, an abnormality was seen exclusively on the CECT in 3 out of 547 cases $(0.5 \%)$ and it changed the diagnosis in 15 cases $(2.7 \%)$. The clinicians in this study had some limited access to an MRI and patients with demyelinating diseases, cranial nerve pathology, sella pathology, infection, metastasis and neoplasm were excluded from CT. In our setting, these patients would all receive a cranial CT before a MRI would be considered. This study suggests that taking the clinical history into consideration when deciding whether a CECT is indicated can reduce the risk of missing abnormal findings, although the number of patients in the group with lateralising signs and normal NECT was small. Comparing the results from the study conducted by Chishti et al. ${ }^{1}$ to the results from the study reported here, despite marked differences in the methodology, both showed a very small prevalence of abnormal findings undiagnosed on NECT. Both studies also suggest that the risk can be reduced if history is taken into account when the decision is made whether to do a CECT or not.

Wood et al. ${ }^{11}$ evaluated the value of CECT in the nontrauma patient in the emergency room. Only 3 out of 240 cases $(0.0125 \%)$ had abnormalities on the CECT not initially evident on the NECT. In their study, the additional information did not alter patient management. They concluded that in the acute setting if an NECT is normal the CECT is usually not necessary. ${ }^{11}$ The results of the study by Wood et al. ${ }^{11}$ correlate with the study reported here although they reported fewer cases with additional findings on the CECT. This could probably be attributed to the higher tuberculosis and HIV burden in the South African population. 
Radiation exposure is an important risk factor to consider when performing a CT of the brain. Ionising radiation has many documented adverse effects. The most serious is the induction of cancer. Cancer is a complication of radiation and has no threshold dose, thus even a small dose will have a small risk. The CT dose from each CT is cumulative. A recently published retrospective cohort study by Pearce et al. on radiation exposure from CT scans in childhood showed a positive association between the radiation dose from CT scans and the development of leukaemia and brain tumours. ${ }^{12}$ The study estimates an excess relative risk (ERR) of 0.036 per $m G y$ (CI 0.005-0.120; $p=0.0097$ ) for leukaemia and an ERR of 0.023 per mGy (CI 0.010-0.049; $p<0.0001$ ) for brain tumours following childhood exposure. The estimated brain dose received from a brain CT in the study by Pearce et al. ${ }^{12}$ ranged between 28 and $44 \mathrm{mGy}$ and the estimated red marrow dose ranged between 2 and 9 mGy. In the study by Pearce et al., cumulative doses of 50 mGy almost tripled the risk for leukaemia and cumulative doses of $60 \mathrm{mGy}$ tripled the risk for brain tumours. ${ }^{12}$ The effective dose of a brain CT is approximately $4 \mathrm{mSv}$, which is equivalent to 200 chest radiographs. ${ }^{13}$ A pre-contrast and post-contrast $\mathrm{CT}$ result in double the radiation dose and would therefore carry double the risk for development of cancer. Radiologists must keep radiation exposure as low as reasonably achievable (the ALARA principle). This is another reason for re-evaluating CT protocol.

An important consideration is the risk of adverse reactions related to intravenous contrast media used in CECT. As with any drug, intravenous iodinated contrast media have a risk of adverse reactions. These can be divided into general and organ-specific reactions including nephro-, cardiovascular, pulmonary and neurotoxicity. ${ }^{14,15}$

General reactions can be subdivided into acute and delayed reactions, whilst acute reactions can be subdivided into mild, moderate and severe reactions. Mild reactions (nausea, vomiting, limited urticaria, extremity pain) have a reported incidence of $3 \%$ when using non-ionic intravenous contrast media (NICM). Moderate reactions (severe vomiting, extensive urticaria, dyspnoea, rigors and laryngeal oedema) have a reported incidence of $0.2 \%-0.4 \%$ and severe reactions (pulmonary oedema, hypotension, loss of consciousness, cardiac arrest and arrhythmias) are seen in $0.04 \%$ of NICM. $^{14,15}$ A review of 48 fatal reactions revealed renal failure $(58 \%)$ as the most common cause of death followed by anaphylaxis and allergy (19\%). ${ }^{16}$ Cardiopulmonary arrest $(10 \%)$, respiratory failure $(8 \%)$, cerebrovascular incidents and hypoxia (4\%) accounted for a quarter of deaths. ${ }^{16}$ Contrastinduced nephropathy (CIN), the acute decline in renal function within 72 hours of intravenous contrast injection, is the most common organ-specific adverse reaction. Patients who develop CIN have an increased risk of developing chronic renal disease. ${ }^{17}$

In the developing world, the cost of contrast media is always a factor that needs consideration. The cost of contrast media, the operational cost of the CT scanner and the number of CT scans performed per day. Throughput of patients can be increased if either NECT or CECT were performed. This would in turn also reduce the cost involved to both patient and department. The cost reduction includes servicing and electricity, radiographer time and contrast media costs.

\section{Conclusion}

In many centres in South Africa, CT remains the first-line diagnostic imaging investigation for suspected intracranial pathology. A diagnostically sound yet cost-effective approach to brain CT is required. The risk to the patient from iodinated intravenous contrast media and radiation exposure should be minimised but weighed against the risk of missing pathology.

In this study, only $3.28 \%$ of NECT scans had findings undiagnosed by three reviewers, with a reader error of $1.85 \%$. It was noted retrospectively that $1.42 \%$ of cases had an abnormality only visible on CECT.

The radiation exposure and operational cost could be reduced and patient throughput increased by omitting either NECT or CECT. The risk of moderate and severe contrast-mediainduced adverse events is only slightly smaller than the prevalence of missing abnormal findings on a normal NECT. The possibility of missing occult findings could be reduced by assessing the history and evaluating the indications for a CECT.

Patients with fever, meningism, confusion, focal signs and a history suggestive of vascular lesions or risk factors for dural venous sinus thrombosis should have a CECT only. In patients with a normal NECT and none of these indications there is a small risk of missing abnormal findings on NECT, and CECT could therefore be omitted.

Doing only an NECT in carefully selected cases would reduce the total amount of iodinated intravenous contrast used in the department and thus reduce the cost with a small risk of missing occult pathology. The alternative of performing only a CECT would reduce the risk of missing pathology and reduce the radiation burden. This study, however, did not evaluate the use of CECT without performing NECT. A further study evaluating the advantages and disadvantages of only a CECT would be of value.

\section{Acknowledgements}

The authors wish to thank H.S. Schoeman (University of Limpopo) and D. van Zyl (University of Pretoria) for conducting the statistical analysis.

\section{Competing interests}

The authors declare that they have no financial or personal relationship(s) that may have inappropriately influenced them in writing this article. 


\section{Authors' contributions}

C.M. (University of Limpopo) was the lead researcher and was responsible for the concepts, design, literature review and preparation of the manuscript. C.M. (University of Limpopo), F.E.S. (University of Pretoria) and N.Z.M. (University of Pretoria) conducted the study. M.E.K. (University of Limpopo) and N.E. (University of Limpopo) made conceptual contributions and were involved with the manuscript editing.

\section{References}

1. Chishti FA, Al Saeed OM, Al Khawari H, Shaikh M. Contrast-enhanced cranial computed tomography in magnetic resonance imaging era. Med Princ Pract. 2003;12:248-251. http://dx.doi.org/10.1159/000072292

2. Huckman MS. Clinical experience with the intravenous infusion of iodinated contrast material as an adjunct to computed tomography. Surg Neurol. 1975;4:297-318.

3. Cowan I, MacDonald S. How useful is contrast enhancement after a normal unenhanced computed tomography brain scan? Australas Radiol. 1999;43: 448-450. http://dx.doi.org/10.1046/j.1440-1673.1999.00710.x

4. Albertyn LE. Rationales for the use of intravenous contrast medium in computed tomography. Australas Radiol. 1989;33:29-33. http://dx.doi.org/ 10.1111/j.1440-1673.1989.tb03231.x

5. Kramer RA, Janetos GP, Perlstein G. An approach to contrast enhancement in computed tomography of the brain. Radiology. 1975;116:641-647.
6. Bernard MS, Hourihan MD, Adams H. Computed tomography of the brain: Does contrast enhancement really help? Clin Radiol. 1991;44:161-164. http://dx.doi. org/10.1016/S0009-9260(05)80860-8

7. Barrington NA, Lewtas NA. Indications for contrast medium enhancement in computed tomography of the brain. Clin Radiol. 1977;28:535-537. http://dx.doi. org/10.1016/S0009-9260(77)80073-1

8. Butler AR, Kricheff II. Noncontrast CT scanning: Limited value in suspected brain tumour. Radiology. 1978;126:689-693.

9. European Society of Radiology [homepage on the internet]. Risk management in radiology in Europe IV. 2004 [cited 2011 January 09]. Available from: http://www. myesr.org/html/img/pool/ESR_2006_IV_Riskmanagement_Web.pdf

10. Royal College of Radiologists. Risk management in clinical radiology. London: Royal College of Radiologists; 1995.

11. Wood LP, Parisi M, Finch IJ. Value of contrast enhanced CT scanning in the nontrauma emergency room patient. Neuroradiol J. 1990;32:261-264. http://dx.doi org/10.1007/BF00593043

12. Pearce MS, Salotti JA, Little MP, et al. Radiation exposure from CT scans in childhood and subsequent risk of leukemia and brain tumours: A retrospective cohort study. Lancet. 2012;380:499-505. http://dx.doi.org/10.1016/S0140-6736(12)60815-0

13. Shah NB, Platt SL. ALARA: Is there a cause for alarm? Reducing radiation risk from computed tomography scanning in children. Curr Opin Pediatr. 2008;20:243-247. http://dx.doi.org/10.1097/MOP.0b013e3282ffafd2

14. Namasivayam $S$, Kalra MK, Torres WE, et al. Adverse reactions to intravenous iodinated contrast media: A primer for the radiologist. Emerg Radiol. 2006;12: 210-215. http://dx.doi.org/10.1007/s10140-006-0488-6

15. Namasivayam S, Kalra MK, Torres WE, et al. Adverse reactions to intravenous iodinated contrast media: An update. Curr Probl Diagn Radiol. 2006;35:164-169. http://dx.doi.org/10.1067/j.cpradiol.2006.04.001

16. Wysowski DK, Nourjah P. Deaths attributed to $x$-ray contrast media on US death certificates. Am J Roentgenol. 2006;186:613-615. http://dx.doi.org/10.2214/ AJR.04.1790

17. Cronin RE. Contrast-induced nephropathy: Pathogenesis and prevention. Pediatr Nephrol. 2010;25:191-204. http://dx.doi.org/10.1007/s00467-009-1204-z 